\title{
Calculation Of Atmospheric Neutrino Flux Based On AMS02 Observations
}

\section{Morihiro Honda*Institute for Cosmic Ray Research, University of Tokyo, Kashiwa, Chiba, 277-8582 Japan.}

E-mail: mhondadicrr.u-tokyo.ac.jp

\section{Takaaki Kajita}

Institute for Cosmic Ray Research, University of Tokyo, Kashiwa, Chiba, 277-8582 Japan.

E-mail: Kajitadicrr.u-tokyo.ac.jp

\section{Katsuaki Kasahara}

Research Institute for Science and Engineering, Waseda University, Tokyo, 169-8555, Japan.

E-mail: kasaharadicrr.u-tokyo.ac.jp

\section{Shoichi Midorikawa}

Faculty of Engineering, Aomori University, Aomori, 030-0943 Japan.

E-mail: midorieaomori-u.ac.jp

\section{Jun Nishimura}

Institute of Space and Astronomical Science, JAXA, Sagamihara, Japan

E-mail: ANB05012@nifty.com

The primary flux model for the calculation of atmospheric neutrino is renewed based on the AMS02 observation of primary cosmic rays. With new cosmic ray spectra model, the interaction model has been revisited, so that it reproduce the observed muon flux accurately. Then, we applied the primary cosmic ray flux model and the interaction model to the calculation of atmospheric neutrino flux. In this paper, some of preliminary results are presented.

35th International Cosmic Ray Conference - ICRC2017

10-20 July, 2017

Bexco, Busan, Korea

\footnotetext{
* Speaker.
} 


\section{Introduction}

Recently there have been important progresses in the observation of cosmic rays achieved by AMS02 [四]. AMS02 has been observing the primary cosmic ray on ISS (International Space Station) [ [] ] since November 2011, equipped with a large magnetic spectrometer, and have compiled first 2 years data for the energy spectra of primary cosmic ray protons and heliums up to a little above $1 \mathrm{TeV}$, overwhelming other experiments in the precision and statistics. We construct a new primary cosmic ray spectra model based on the AMS02 observation. Above $10 \mathrm{TeV}$, although the observation of AMS02 does not reach to this energy region yet, it works as a guide to select useful data from the balloon borne experiments in this energy region.

Even the maximum energies of observed cosmic rays are lower than that of AMS02, there are some important cosmic ray observation experiments such as the series of BESS [四], BESS$\mathrm{TeV}$ [], BESS-Polar [ [0], and the PAMELA [ []] experiments. They are equipped with magnetic spectrometers, and their results below $\sim 100 \mathrm{GeV}$ agree well with AMS02. They provided us useful information especially on the solar modulation of cosmic rays, which is important in the "muon calibration" of the hadronic interaction model.

In our previous works [8], we constructed the primary spectra model based on AMS01 [3] and BESS [四] observations. Above a few $100 \mathrm{GeV}$, we assumed a simple power law spectra, and the power is determined by the data from balloon borne experiments above $10 \mathrm{TeV}$. With this primary cosmic ray spectra model, we modify the hadronic interaction model, so that it reproduces the observed muon spectra. We call this procedure as the muon calibration. The muon calibration has to be repeated also for the primary cosmic ray spectra model constructed here.

With the primary cosmic ray spectra model based on the AMS02 observation, and with muon calibrated hadronic interaction model, we calculate the atmospheric neutrino flux at Kamioka, and compare it with previously calculated results for the all direction average and in the average in 5 zenith angle bands. Note, these results are still preliminary mainly due to small statistics.

\section{Cosmic Ray Spectra Model and AMS02 Observation}

In the left panel of Fig. W, we depicted the observed cosmic ray spectra, considered in the construction of our new cosmic ray spectra model. One of the most important features in the cosmic ray spectra reported by AMS02 is the bending at $\sim 500 \mathrm{GeV}$ for protons, and at $\sim 250 \mathrm{GeV} / \mathrm{n}$ for heliums. As is seen in the left panel of Fig. W, the line drawn from the bending to the center of the selected proton data from the balloon borne experiments at around $10 \mathrm{TeV}$ roughly agrees with the AMS02 proton spectrum above the bending. However, the helium spectra observed by balloon borne experiment above $1 \mathrm{TeV}$ show large differences among them, even when their proton spectra agree with each other. We selected the data most agreed with the extension of AMS02 helium spectrum.

For the cosmic ray protons, all the balloon borne experiments show a breakdown of spectra at around $30 \mathrm{TeV}$. Therefore, we assumed the second bending or the change of the spectral index at around $30 \mathrm{TeV}$ for protons. For the cosmic ray heliums, we assumed the second bending at around $15 \mathrm{TeV} / \mathrm{n}$, accordingly. However, the change of spectral index suggested by the balloon 

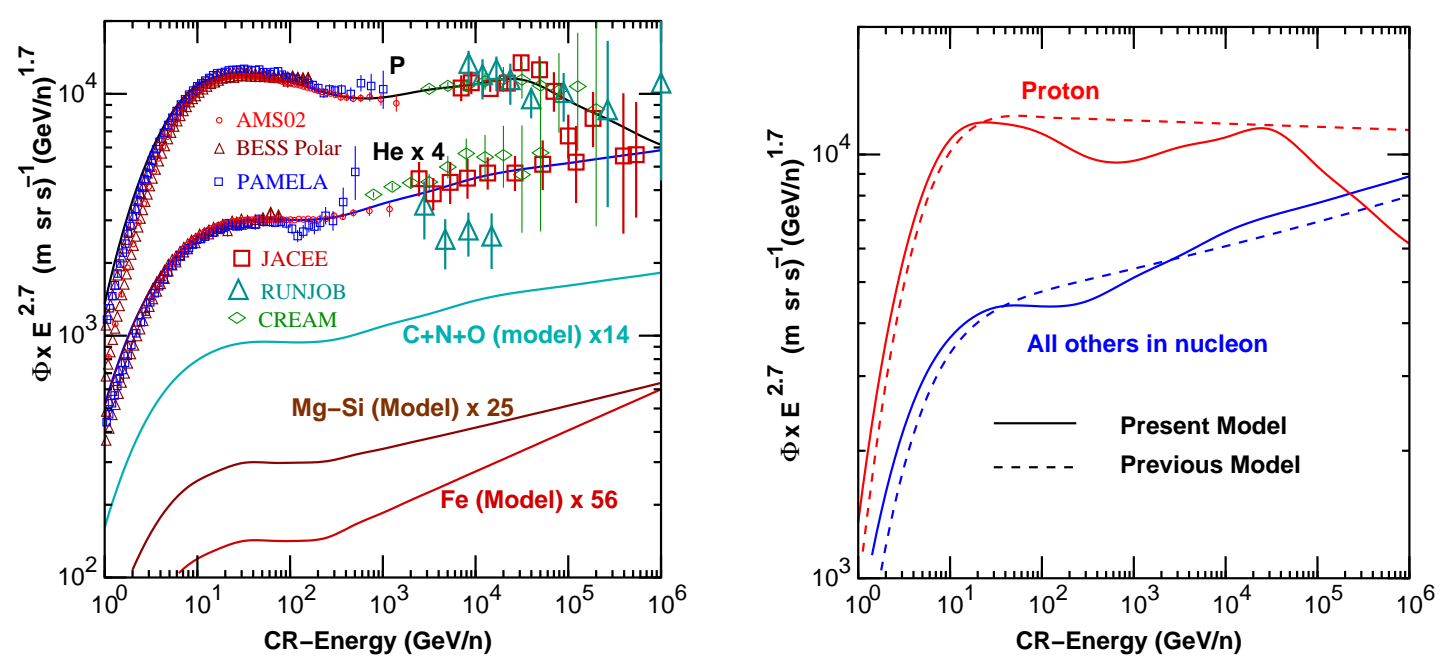

Figure 1: Left panel: The cosmic ray spectra data for protons and Heliums. AMS02 is from Ref [山],

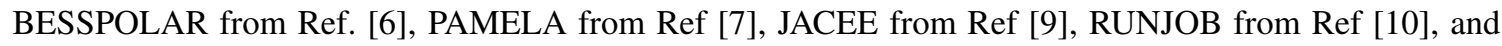
CREAM from Ref [W]]. Right panel: the temporal cosmic ray spectra model for cosmic ray protons and Heliums, and the data used in the model construction. The dashed lines show the previous primary cosmic ray spectra model.

borne experiments is smaller than that of cosmic ray protons. Hereafter, we call thus constructed primary cosmic ray spectra model as the new primary cosmic ray spectra model.

In the right panel of Fig. 四, we depicted our primary spectra model for proton and all other nucleon spectra. The new primary cosmic ray spectra model gives smaller cosmic ray proton flux above a few $10 \mathrm{GeV}$ except for at around $20 \mathrm{TeV}$. The decrease from previous model is $\sim 30 \%$ at around $1 \mathrm{TeV}$. On the other hand the cosmic ray helium spectrum shows a decrease of $\sim 15 \%$ at around $200 \mathrm{GeV} / \mathrm{n}$, but shows a increase of $\sim 10 \%$ above $10 \mathrm{TeV} /$. The spectrum of cosmic ray helium is much closer to that of previous cosmic ray spectra model.

In the energy region above $100 \mathrm{TeV}$, the direct measurement of cosmic ray is difficult yet, and cosmic ray spectra are considered to have a complex structure called "knee". However, the variation of cosmic ray spectra above $100 \mathrm{TeV}$ gives small effects on the atmospheric neutrino below $100 \mathrm{GeV}$, which is the main target in our calculation of the atmospheric neutrino flux. Here, we stop the construction of cosmic ray spectra model at $100 \mathrm{TeV}$, and just show the extension to $1 \mathrm{PeV}$ in the figure.

\section{Solar Modulations Of Cosmic Rays}

Before we start the muon calibration, we have to know the precise primary cosmic ray spectrum at the observation date. Therefore, we study the Solar modulation of primary cosmic rays in advance using recent cosmic ray data.

As the measure of the Solar activity, the count of neutron monitor is generally used. The neutron monitor is the detector to count the neutron in the air, which is created in the hadronic interaction of cosmic rays and the air nucleus. Thus the variation of the count of neutron monitor is directly connected to the variation of primary cosmic rays flux. We show the count of neutron 


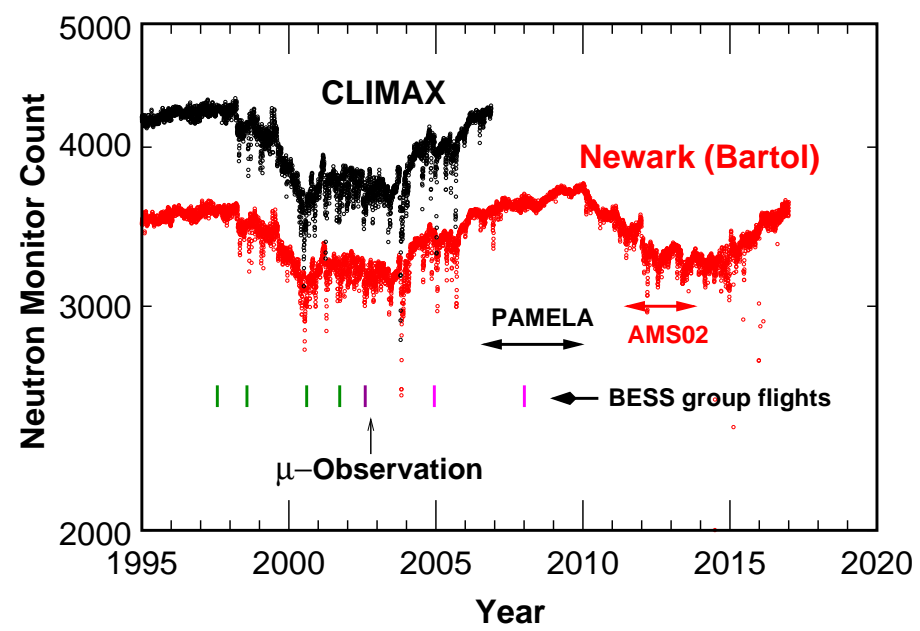

Figure 2: The count of neutron monitor at Climax and Newark with the dates of cosmic ray observations.
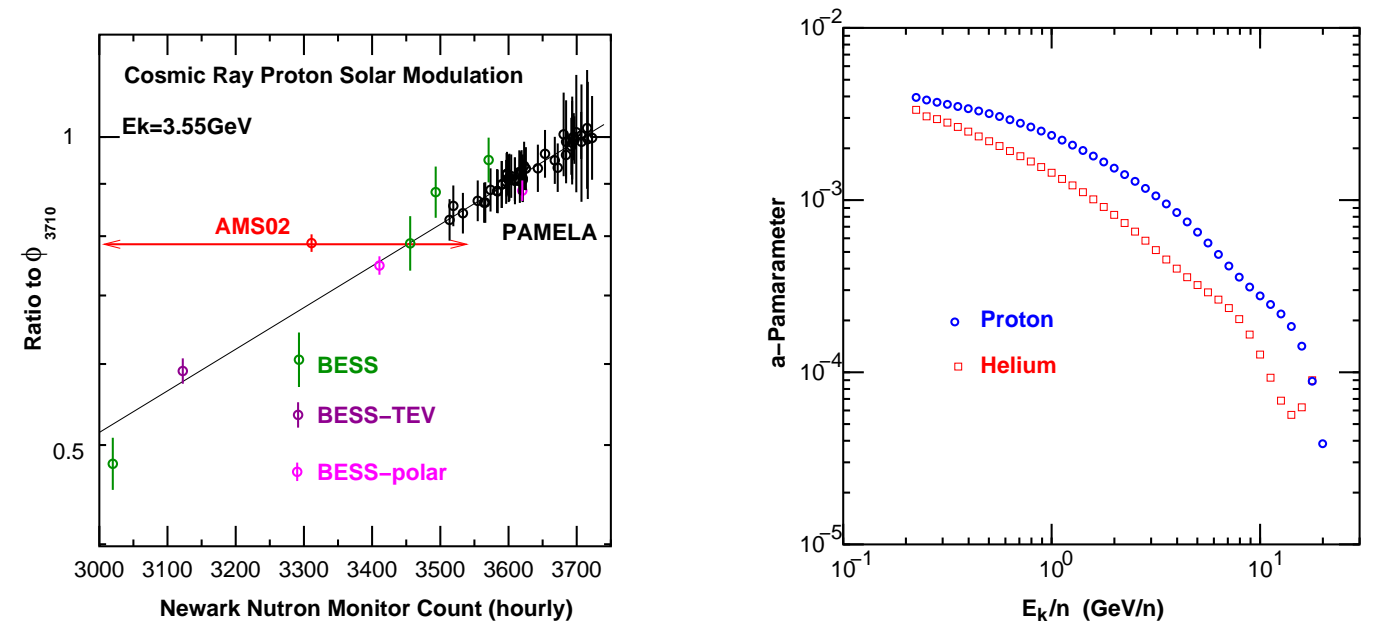

Figure 3: Left panel: observed cosmic ray proton spectra at $3.55 \mathrm{GeV}$ by different experiments. Right panel: $a$-parameter in eq. 3.1 at each energy determined by the least mean square method using the observed cosmic ray spectra.

monitor at Climax [[13] and Newark [14]] in Fig. \], with the operation date of several cosmic ray observation experiments. Note, although the data of Climax neutron monitor was used to study the Solar modulation of cosmic rays for a long time, it was not updated since 2007. we use the Newark neutron monitor data in this study.

For the cosmic ray data, we use the data from BESS group (BESS, BESS-TEV, BESS-Polar) and PAMELA monthly data [[5]. in this study. PAMELA had observed the cosmic rays from July 2006 to January 2010, and analyzed the cosmic ray data monthly for cosmic ray protons. We plotted the cosmic ray proton flux at $E_{k i n}=3.55 \mathrm{GeV}$ from those cosmic ray observation experiments in left panel of Fig. [1], as the function of neutron monitor count at Newark. We find the BESS group data are well spreaded in the variation range of the neutron monitor count. On the other hand, the observation period of PAMELA is close to the minimum activity phase of Solar cycle, and the neutron monitor counts are close to the its maximum value. This data is useful to study the Solar 
modulation at the phases close to the activity minimum in detail, but not to study it at the higher activity phases of Sun.

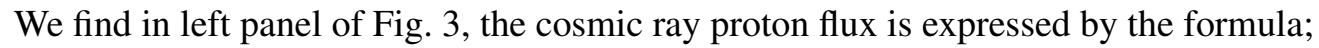

$$
\phi(E, N)=\left\{\begin{array}{l}
\phi(E, 3710) \exp (a \cdot(N-3710)) \\
\phi(E, 3710)
\end{array}\right.
$$

where $N$ is the neutron monitor count at Newark, and $a$ is a constant determined for each cosmic ray energy. $N=3710$ was not the maximum count in the period of PAMELA observation, but the increase of cosmic ray spectra was saturated at this count. It is noted that the PAMELA monthly data below $N=3710$ also follow eq. B.] well.

We determine the parameter $a$ by the least mean square method for cosmic ray protons and heliums from $0.2 \mathrm{GeV} / \mathrm{n}$ to $20 \mathrm{GeV} / \mathrm{n}$, and plotted in the right panel of Fig. [1. We use eq. [.] and the $a$ parameter determined here to estimate the Solar modulated primary cosmic ray spectra. Practically we can consider that the cosmic ray protons with energy $\gtrsim 18 \mathrm{GeV}$, and the cosmic ray heliums with energy $\gtrsim 12 \mathrm{GeV} / \mathrm{n}$ are free from the Solar modulation.

We note, AMS02 has started the observation on November 19, 2011 and continue to date, and compiled the data until November 26, 2013 into cosmic ray proton and helium spectra. The observation period for those spectra is shorter than that of PAMELA, but the variation of neutrino monitor count is larger than that of PAMELA. If they analyze the data in shorter period as PAMELA did, it would largely improve the study of Solar modulation of cosmic rays.

\section{Muon Calibration}
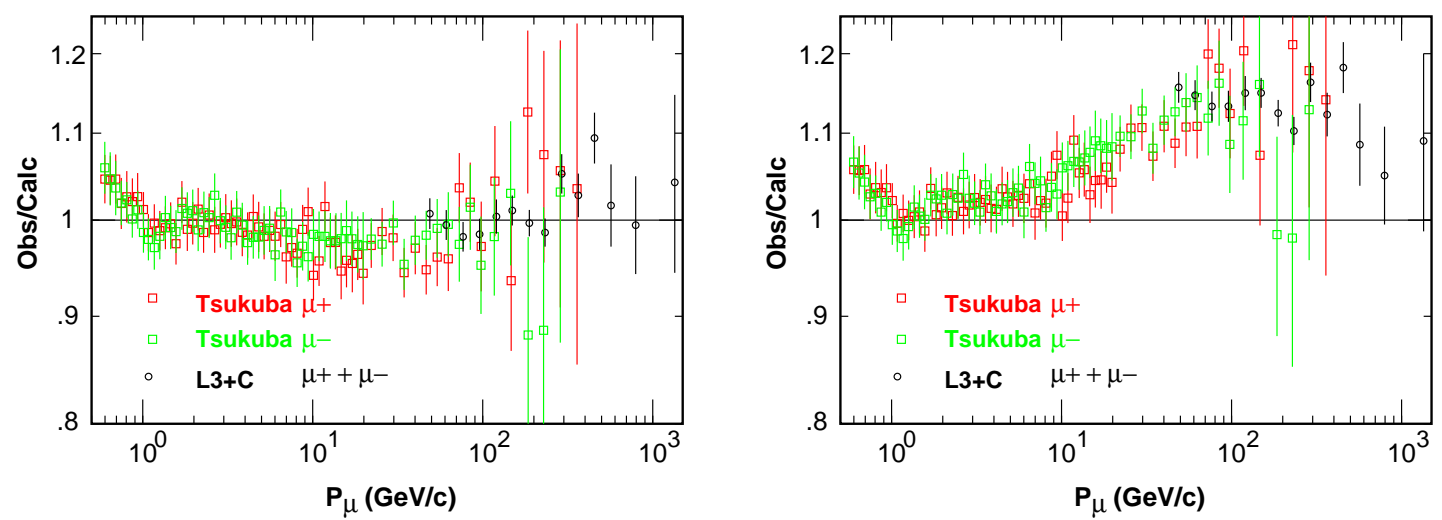

Figure 4: Comparison of the observed muon spectra and calculated ones in the ratio. In the left panel, we used previous primary cosmic ray spectra model and the interaction model calibrated with the muon data and previous primary cosmic ray spectra model. In the right panel, we used the same interaction model as left panel and the primary flux spectra model based on the AMS02 observations explained in this work.

In the muon calibration, we use the data from 2 muon observation experiments. One is that carried out by the BESS group using the BESS-TeV equipment from December 1, 2002 to December 6, 2002 at Tsukuba [5]. The average neutron monitor count of Newark in this observation 


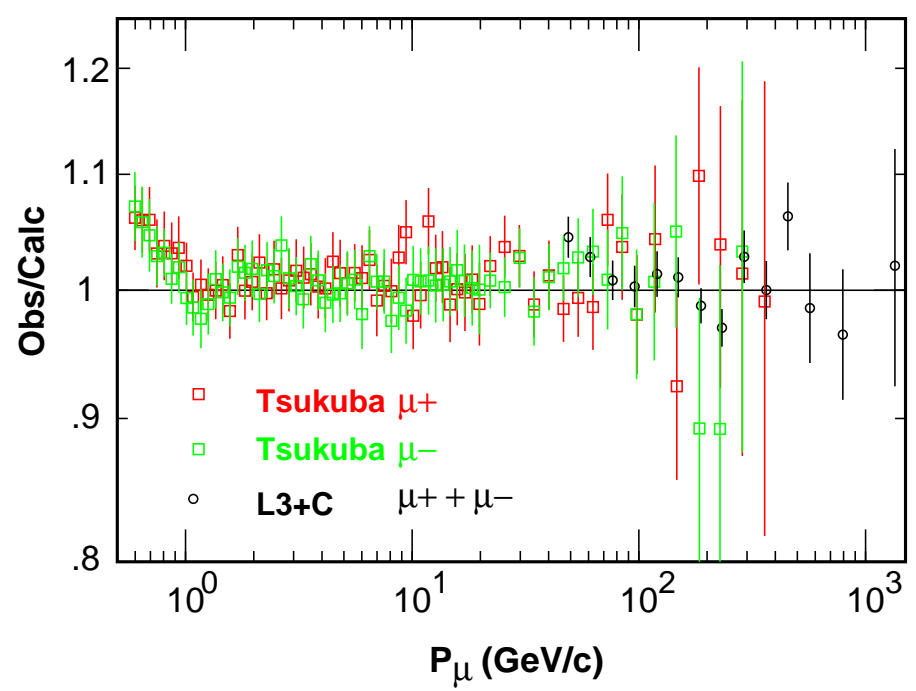

Figure 5: Comparison of the observed muon spectra and calculated ones in the ratio. We used the new primary cosmic ray spectra model and muon calibrated hadronic interaction model with it.

period was 3156. The other is date from the $\mathrm{L} 3+\mathrm{C}$ experiment [16] with trigger and data acquisition system independent of L3 detector in the years 1999 and 2000 at CERN. However, as the momentum of muons observed by $\mathrm{L} 3+\mathrm{C}$ is high enough, and are free from the Solar modulation or rigidity cutoff.

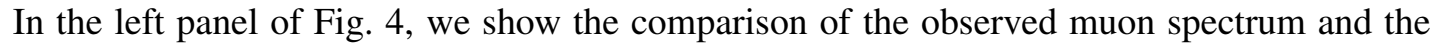
calculated muon spectra with our previous interaction model and with our previous primary cosmic ray spectra model. As the hadronic interaction model was calibrated with the observed muon spectra using previous cosmic ray spectra model, we find a good agreement in this comparison. In the right panel of Fig. 团, we show the same comparison, but we used our previous interaction model and the new primary cosmic ray spectra model explained in the sec. $\square$ in the calculation of muon spectra. We find 10-15\% deficits in the calculated muon spectra at $30-300 \mathrm{GeV} / \mathrm{c}$ in the right panel of Fig. 田. We note that the Solar modulation is taken into account to both cosmic ray spectra following the discussion in sec. [3.

These deficit in the calculated muon spectra is recovered by a hardening of secondary pion spectrum of the hadronic interactions between the cosmic rays and air nuclei. Such a hardening of secondary spectrum is easily done in our inclusive interaction model, also modifying other secondary spectra to maintain the energy conservation in average. In Fig. [], we show the comparison of observed and calculated muon spectra with new primary cosmic ray spectra model and muon calibrated hadronic interaction model with it. Comparing the results of old and new muon calibrations, we find the agreement is better in new muon calibration (Fig. (1) than the old one (left panel of Fig. (1). This is because we had tried to modify the hadronic interaction model as little as possible, leaving the residuals of the reconstruction as large as the experimental error, in the old muon calibration. On the other hand we tried to reconstruct the observed muon spectra as much as possible in the new muon calibration.

We use thus muon calibrated hadronic interaction model as in Fig. $\$$ in the calculation of atmospheric neutrino flux with the new primary cosmic ray spectra model. 


\section{Atmospheric Neutrino Flux}
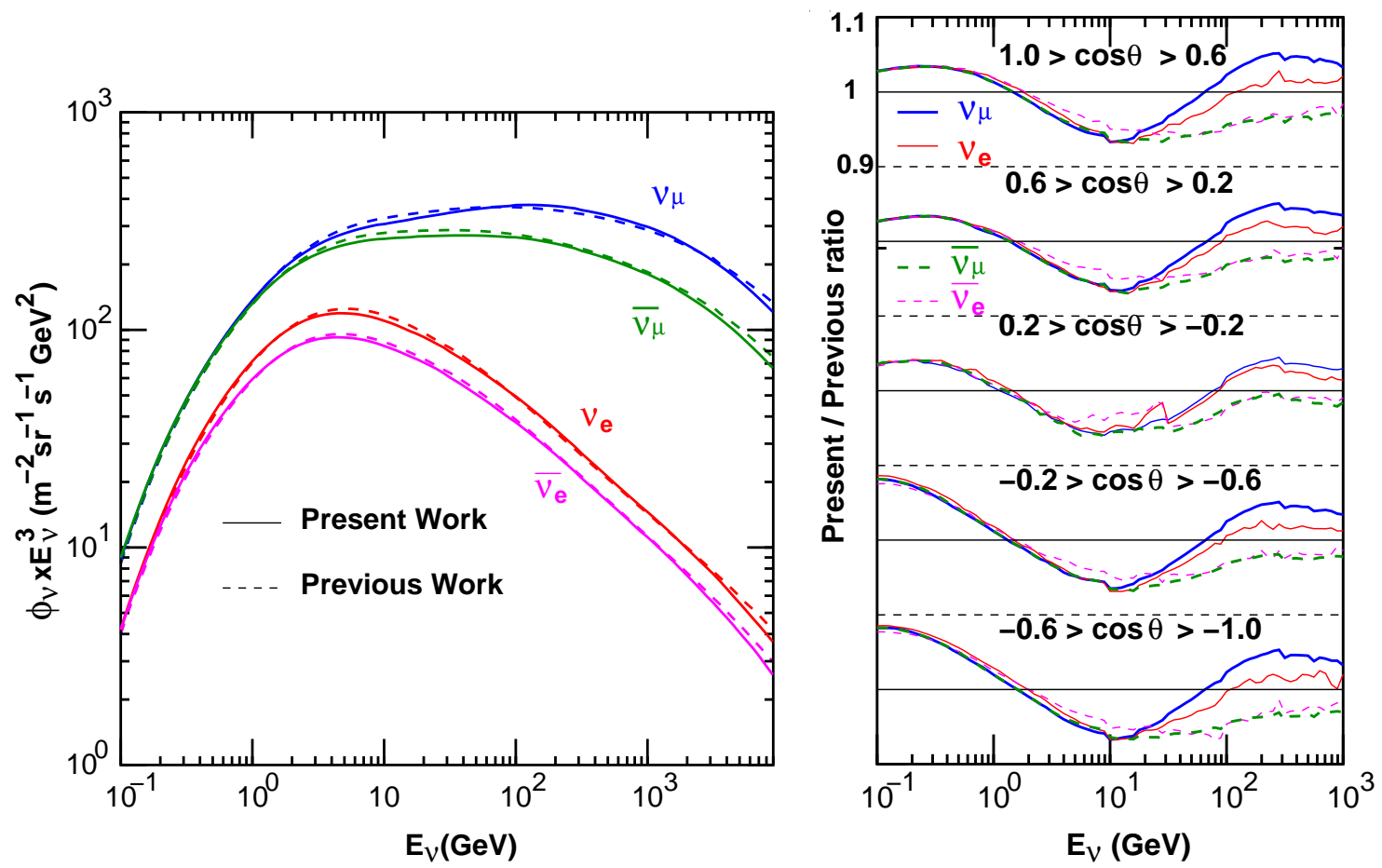

Figure 6: Left panel: The atmospheric neutrino flux averaged over all directions for Kamioka site with the primary spectra model based on the AMS02 observation with the muon calibrated interaction model with it. For the comparison, we also depicted the atmospheric neutrino flux calculated in the previous work in dashed line. Right panel: The ratio of present to previous atmospheric neutrino fluxes at Kamioka, averaging them in 5 zenith angle bins.

In this section, we show the preliminary results of new calculation of the atmospheric neutrino fluxes, with the new primary cosmic ray spectra model and the muon calibrated hadronic interaction model with it, for Kamioka, and compare the new calculation with the previous calculation [ए2].

In left panel of Fig. G, we depicted the atmospheric neutrino flux in the new calculation averaged over all directions and at Kamioka. In the same figure, we also depicted the same quantities in the previous calculation. As is seen in this figure that over all feature is similar to each other, but there is a clear deficit of neutrino flux at around $10 \mathrm{GeV}$ in the new calculation for all 4 flavors. However, at around $100 \mathrm{GeV}$, the fluxes in both calculations agree well with each other.

To magnify the difference, and to check the zenith angle dependence, we calculate the average of the atmospheric neutrino flux in 5 zenith angle bins, and plotted the new/old ratio in the right panel of Fig. 6. Although the statistics is not enough yet, especially at near horizontal directions, we find the deficit of a little more than $5 \%$ in the new calculation at around $10 \mathrm{GeV}$ for all zenith angle bins, and for all neutrino species. At high energies $(100 \gtrsim \mathrm{GeV})$, we find a little increase of $v_{\mu}$ and $v_{e}$ fluxes in new calculation, and a little decrease of $\bar{v}_{\mu}$ and $\bar{v}_{e}$ fluxes. However, the difference could be explained by the difference between old and new muon calibrations.

At the lower energy below $1 \mathrm{GeV}$, we find new calculation show the increase of neutrino flux close to $10 \%$ as energy decrease. We note below $1 \mathrm{GeV}$ is outside of valid region of the muon 
calibration using the muon data observed at sea level. However, we find the difference of old and new calculations is well less than $10 \%$ from $0.1 \mathrm{GeV}$ to $1 \mathrm{TeV}$, and at most energies, the difference is less than $5 \%$. Therefore, the zenith angle dependence of both calculations are very similar to each other.

\section{Summary}

We have constructed new primary cosmic ray spectra model based on AMS02 for our atmospheric neutrino flux calculation. Also the Solar modulation is studied with the cosmic ray observations of BESS group, and the monthly spectra by PAMELA observation. The hadronic interaction model is tuned with the observed muon spectra data and with above study.

Then we calculate the atmospheric neutrino flux and compared with the one calculated in the previous work. The atmospheric neutrino flux in new calculation generally agrees with that of previous calculation. However, there should be a certain decrease of neutrino flux at around $10 \mathrm{GeV}$ almost independently of neutrino flavors and directions.

\section{Acknowledgments}

We are grateful to M.S. Athar and S. Haino for the discussions. We also thank the ICRR of the University of Tokyo, especially for the use of the computer system.

\section{References}

[1] M. Aguilar, et al. (AMS02) Phys. Rev. Lett., 114, (2015) 171103, and Phys. Rev. Lett., 115, (2015) 211101.

[2] see https://www.nasa.gov/mission_pages/station/main/index.html.

[3] J. Alcaraz et al. (AMS-01), Phys. Lett. B490, (2000) 27.

[4] T. Sanuki et al. (BESS), Astrophys. J. 545, (2000) 1135.

[5] S. Haino et al. (BESS-TeV), Phys. Lett. B 594, (2000) 35.

[6] K. Abe et al., arXiv:1506.01267 [astro-ph.HE]

[7] O. Adriani et al. (PAMELA), Science 332, (2011) 69,

[8] M. Honda et al., Phys. Rev. D 70, (2004) 043008.

[9] K. Asakimori et al., (JACEE) Astrophys. J. 502, (1998) 278.

[10] A.V. Apanasenko et al., (RUNJOB) Astropart. Phys. 16, (2001) 13.

[11] Y.S Yoon et al. (CREAM-I), Astrophys.J. 728, (2011) 122.

[12] M. Honda et al., Phys. Rev. D 92, (2015) 023004.

[13] see http://cr0.izmiran.rssi.ru/clmx/baseinfo.htm

[14] see http://neutronm.bartol.udel.edu/

[15] see https://lpsc.in2p3.fr/cosmic-rays-db/

[16] P. Achard et al. (L3+C), Phys. Lett. B598, 15 (2004). 